\title{
A Study of Lattice Elasticity from Low Entropy Metals to Medium and High Entropy Alloys
}

\author{
E-Wen Huang ${ }^{1, *}$, Dunji $\mathrm{Yu}^{2}$, Jien-Wei Yeh ${ }^{3}$, Chi Lee $^{3}, \mathrm{Ke} \mathrm{An}^{2}$, and Shan-Yi Tu ${ }^{1,4}$ \\ ${ }^{1}$ Department of Materials Science and Engineering, National Chiao Tung University, \\ 1001 University Road, Hsinchu, Taiwan 30010, Taiwan (R.O.C.) \\ ${ }^{2}$ Chemical and Engineering Materials Division, the Spallation Neutron Source, Oak \\ Ridge National Laboratory, Oak Ridge, TN 37831, United States \\ ${ }^{3}$ Department of Materials Science and Engineering, National Tsing Hua University, \\ Hsinchu, 30013, Taiwan (R.O.C.) \\ ${ }^{4}$ Department of Chemical and Materials Engineering, National Central University, \\ Jhongli, 32001, Taiwan (R.O.C.)
}

\begin{abstract}
An equal-molar CoCrFeMnNi, face-centered-cubic high-entropy alloy system and a face-centered-cubic stainless steel described as a medium-entropy system, are measured by in-situ neutron-diffraction experiments subjected to continuous tension at room and several elevated temperatures, respectively. With spallation neutron, the evolution of multiple diffraction peaks are collected simultaneously for lattice-elasticity study. Temperature variation of elastic stiffness of a single face-centered-cubic-phase $\mathrm{Ni}$ and a single face-centered-cubic-phase Fe are compared as low-entropy metals. The CoCrFeMnNi high-entropy alloy show distinct lattice anisotropy.
\end{abstract}

Keywords: neutron diffraction, metal and alloys, elastic behavior, microstructure, high entropy alloy 
In 2014, Gludovatz et al. [1] report superior fracture-resistant property of an equiatomic, face-centered-cubic, CoCrFeMnNi-high-entropy alloy (HEA) for cryogenic applications. Moreover, several nontrivial mechanical behavior of this kind of HEA is observed by Yeh et al. [2], Nieh et al. [3] and George et al. [4], respectively. Besides its practical application [1], the underneath mechanical mechanisms of this CoCrFeMnNi-high-entropy alloy are unclear. For example, how equiatomic-element mismatch affect its bonding [5]? Would Vegard's law be valid [6]?

For metallic systems, numerous work have demonstrated the importance of ordering for compositions [7, 8]. Comparing with the metallic systems, Ashyby's summarizes the basic mechanical and thermal properties of materials and reveals the sub-range associated with each material class [9]. Generally, the energy-driven moduli of the soft materials are smaller than their counterparts. The moduli of soft materials typically show temperature-dependent entropy-driven and energy-driven elastic behavior. For the configuration ordering, the entropic elasticity originates from the thermodynamics. When there is configuration rearrangement, the enthalpy of phase transition can well quantify the level of entropic effect after Clausius-Clapeyron relation [10-13]. For the conformation ordering, spatial distribution of a cluster of atoms or molecules brings significant entropy-driven elasticity to local potential energy, too. [14-18] For the metallic systems, such a temperature-dependent moduli subjected to order-disorder transition has also been quantified [19, 20]. For long-range-order-disorder transition, a step-change in Young's modulus of the $\mathrm{Cu}_{3} \mathrm{Au}$ system is observed at critical temperature $[19,20]$. Besides the 
aforementioned long-range-order-disorder transition, there is deformation-induced entropic effect on the modulus found in bulk-metallic-glass (BMG) system [21]. Falk and Langer postulate the dynamics of two-state shear transformation which irreversible motions are governed by local entropic fluctuations in the volumes of the transformation zones at temperatures far below the glass transition. Above all, several results have reported the entropic effect on the elasticity of the metallic systems in terms of the long-range-order-disorder transition $[19,20]$, martensitic transformation [22], viscoplasticity [21], shear transformation [21], and other plasticity-related behavior [23].

Finally, Hufnagel et al.'s in-situ synchrotron diffraction measurements reveal entropic contribution for the stiffness of metallic glasses within elastic limit [24]. Wagner et al.'s molecular dynamics simulation show local elastic properties of a metallic glass [25]. Their results show that the local potential energy can vary significantly in space for non-crystalline materials [24, 25]. However, the BMGs' local modulus distribution is very different from the crystalline with a long-range order. For long-range order existing in space, a unified elastic modulus is expected [25]. Based on such a fact, for polycrystalline metals, the Taylor theory assumes that the strain tensor is symmetrical [26]. Similarly, Molinari et al. use a mean field approach to describe the deformation of polycrystals of an ellipsoidal inclusion embedded in a homogeneous matrix with a unified elastic modulus [27].

In this paper, we focus on $h k l$-dependent elasticity that is the consequence originated from the aforementioned effects. Explicitly, it is an extension of previous 
reports $[4,28]$ which specifies a range of temperature-dependent mechanical-behavior transitions. Otto et al. found strong temperature-dependent behavior on the tensile properties of this system ranging from $77 \mathrm{~K}$ to $1,073 \mathrm{~K}$ [4]. Specifically, Gali and George discovered that the transition between the thermal and athermal regions of the HEAs is higher than that of typical binary alloys [28]. Yeh et al. [2] have concluded such a behavior could reflect the severe distortion in the high-entropy alloys, which creates much more resistance than traditional alloys at the same temperature [29]. The extended X-ray absorption fine structure evidence shows that the lattices of HEAs are distorted by the heterogeneity of local chemical distributions [30]. The aforementioned recent research lead us to ask: could these local compositional heterogeneity of the equiatomic high-entropy alloy be sufficiently enough to break tensor symmetry and collaborate with energy-driven elasticity?

Here we show the equiatomic, face-centered-cubic CoCrFeMnNi metallic system as a high-entropy alloy (HEA); a face-centered-cubic 304L stainless steel (with a weight percentage of C:0.022; Cr:18.10; Ni:8.040; Mn:1.390; Mo:0.550; Cu:0.465; Si:0.256; Co:0.144; N:0.080; W:0.060; V:0.060; P:0.039; S:0.027; Sn:0.015 and balance $\mathrm{Fe}$ ) as a medium-entropy alloy (MEA), and pure $\mathrm{Fe}$ and pure $\mathrm{Ni}$ as low-entropy metals (LEMs). We facilitate the advantage of the in-situ neutron diffraction measurements for its great gauge volume to investigate the ensemble-averaged lattice elasticity of the HEA and the MEA to compare with that of the LEMs. The procedures of in-situ diffraction experiments is described in the session of Methods, which is similar to Wu et al.'s room-temperature measurement 
[31]. The difference is that besides room temperature experiments, we measure both the HEA and MEA at several elevated temperatures as shown in the Supplementary Information. With the event-based data acquisition at Spallation Neutron Source (SNS) of the Oak Ridge National Laboratory (ORNL), neutron diffraction patterns were collected under continuously-loading mode. The feature is especially useful for the current investigation to observe dynamics loading condition [32]. Moreover, the advantage of the spallation-neutron-source materials-engineering diffractometer, such as VULCAN [32] of SNS, is that multiple diffraction peaks are collected simultaneously.

We follow Wu et al.'s presentation [31] to plot elastic modulus of different $h k l$ planes as a function of anisotropy factor $\left(\mathrm{A}_{h k l}\right)$ in Figures $1(\mathrm{a})$ for pure $\mathrm{Ni}, 1(\mathrm{~b})$ for pure Fe, 1(c) for 304L stainless steel, and 1(d) for CoCrFeMnNi HEA, respectively. The definition of $\mathrm{A}_{h k l}$ is shown below.

$$
\mathrm{A}_{h k l}=\frac{h^{2} k^{2}+k^{2} l^{2}+h^{2} l^{2}}{\left(h^{2}+k^{2}+l^{2}\right)}
$$

Figures 1(a) and 1(b) are based on Ledbetter and Reed's temperature-dependent elastic-stiffness-matrix data [33]. We calculate the mechanical elastic constants for its face-centered-cubic structure with Kröner model [34, 35]. Figures 1(c) and 1(d) are our experimental results. Measurements at different temperatures from room temperature to elevated temperatures are labeled in the insets of Figures 1(a) 1(d) accordingly. 
Generally, in Figures 1(a) and 1(b), the moduli and the anisotropy factor $\left(\mathrm{A}_{h k l}\right)$ exhibit a linear relationship. Hutchings et al. [36] have summarized several metallic systems showing the same linearity for their homogenous average elastic properties of the aggregate. Within elastic-deformation limit, there is no plasticity-induced heterogeneous-intergranular stresses. In Figures 1(a) and 1(b), pure Ni and pure Fe show the same feature because their grains are considered chemically and mechanically uniform throughout the aggregate as Taylor [26], Sachs [37], and Kröner [38] constitute.

Besides Hutchings et al.'s [36] and Wu et al.'s [31] presentation for (111), (200), (220), and (311) lattice moduli, in Figures 1(a) 1(d), we add data of (222) and (400) lattice moduli. There is no difference between pure Ni and Pure Fe from (111) and (222) lattice moduli as well as their (200) and (400). It is also not obvious for 304L stainless steel as shown in Figure 1(c). However, the (200) and (400) lattice moduli of the $\mathrm{CoCrFeMnNi} \mathrm{HEA}$ are not identical at Anisotropy factor of zero, especially at 873K. There are also obvious difference between (111) and (222) lattice moduli of the CoCrFeMnNi HEA. Meanwhile, for our CoCrFeMnNi HEA, there are no superlattice peaks showing complete-ordered structure throughout the measurements at various temperature. To further examine the aforementioned phenomenon, we revisit our data and plot the results in Figure 2.

We examine lattice modulus-temperature variation individually as shown in Figures 2(a) for pure Ni, Figure 2(b) for pure Fe, Figure 2(c) for 304L stainless steel, and Figure 2(d) for CoCrFeMnNi HEA, respectively. Their new features 
distinguishing the high-entropy alloys from the others. First, in Figures 2(a) 2(c), the relative variation of lattice moduli decrease almost linearly as temperature increases. In Figure 2(d) at 300K, The relative order of moduli strength follows Generalized Hooke's Law, which is $\mathrm{E}_{111}>\mathrm{E}_{220}>\mathrm{E}_{200}$. However, in Figure 2(d), the (220) shown as $\triangle$ of the CoCrFeMnNi high-entropy alloy do not follow the same trend. At $473 \mathrm{~K}$ and $673 \mathrm{~K}, \mathrm{E}_{200}(\triangle)$ moduli are greater than those of $\mathrm{E}_{111}(\square)$. Moreover, the $\mathrm{E}_{331}(\diamond)$ and the $\mathrm{E}_{420}(\bigcirc)$ also deviate from the room-temperature relative strength order of lattice moduli. Good reported such a moduli transition in the Beta-Brass single crystals which is subjected to an order-disorder transition [39]. However, here is neither phase-transformation-related nor order-disorder-transition-related diffraction-peak evolution seen during our measurements of the current $\mathrm{CoCrFeMnNi}$ high-entropy alloy. The long-range order of the CoCrFeMnNi HEA is invariant as a face-centered-cubic structure throughout the experiments, too. The deviation of $\mathrm{E}_{331}$ $(\diamond), \mathrm{E}_{200}(\triangle)$, and $\mathrm{E}_{420}(\triangle 1$ reflect and return to the room-temperature relative strength order of lattice moduli at $873 \mathrm{~K}$.

On the contrary, from room to high temperatures, the strength of lattice moduli of pure $\mathrm{Ni}$, pure $\mathrm{Fe}$, and 304L stainless steel follow generalized Hooke's law closely. Rollett et al. have discussed the orientation-dependent stored energy [40]. Rollett et al. refer to Rajmohan et al.'s neutron diffraction [41] and Borbély et al.'s x-ray diffraction [42] results as examples, where the manufacturing-induced stored-deformation energy can correlate with Taylor factor. However, for the CoCrFeMnNi high-entropy alloy at $473 \mathrm{~K}$ and $673 \mathrm{~K}, \mathrm{E}_{331}(\diamond), \mathrm{E}_{200}(\triangle)$, and $\mathrm{E}_{420}$ 
( $\square$, show greater moduli. The results of HEA is very different to the others as exampled by Lugovy et al. for elasticity in terms of crystalline tensor [43].

Denton and Ashcroft have discussed the mixture effect on the alloys [6]. They summarize four physical factors including (1) relative atomic sizes; (2) valence electron density; (3) Brillouin-zone effect, and (4) electrochemical differences. The radius ratio between $\mathrm{Cr}, \mathrm{Mn}, \mathrm{Fe}, \mathrm{Co}$, and $\mathrm{Ni}$ in terms of atomic, ionic, covalent, and crystal radii indeed are heterogeneous. Between different pairs of $\mathrm{Cr}, \mathrm{Mn}, \mathrm{Fe}, \mathrm{Co}$, and $\mathrm{Ni}$, the calculated Pauling electronegativities are not uniform, too. Although the atomic numbers of the aforementioned five elements are next to each other, the differences of sizes and individual pair of ionic character do vary, which might correlate with the complicated effect from d-valence electrons.

To compare the bond-length mismatch and elastic properties, Thorpe and Garboczi examined Vegard's law [5]. They propose the bonds have natural lengths $\mathrm{L}_{\mathrm{ij}}^{0}$ with spring constants $\mathrm{K}_{\mathrm{ij}}$. They consider a distribution of random or correlated, of two kinds of bonds. Their model can apply to the current studied face-centered-cubic structure. For this type of problem, Thorpe and Garboczi applied Feynmann-Hellman theorem [44] and stated $(1-\mathrm{x}) K_{A}\left(\left\langle L_{A}\right\rangle-L_{A}^{0}\right)+\mathrm{x} K_{B}\left(\left\langle L_{B}\right\rangle-L_{B}^{0}\right)=0$, where $\mathrm{L}_{\mathrm{A}}^{0}$ and $\mathrm{K}_{\mathrm{A}}$ are the natural length and spring constant for random bonds, respectively, with probability (1-x). Similarly, $\mathrm{L}_{\mathrm{B}}^{0}$ and $\mathrm{K}_{\mathrm{B}}$ are for correlated bonds, respectively, with probability $\quad$ x. $(1-\mathrm{x}) K_{A}\left(\left\langle L_{A}\right\rangle-L_{A}^{0}\right)+\mathrm{x} K_{B}\left(\left\langle L_{B}\right\rangle-L_{B}^{0}\right)=0$ describes that net macroscopic tension is zero relatively to the bonds. However, if the levels of spring constants from random bonds, $\mathrm{K}_{\mathrm{A}}$, and correlated bonds, $\mathrm{K}_{\mathrm{B}}$, are not equal, Thorpe 
and Garboczi conclude that the mean lattice length will not follow Vegard's linear law. The fluctuations originated from bond-length mismatch would "crumple" elastic properties locally.

Referring to the design of the metallic alloys [45-49], the equal-molar $\mathrm{CoCrFeMnNi}$, single face-centered-cubic phase high-entropy alloy can bring in local entropy-driven to the ensemble energy-driven elasticity for the metals. Although experimental results for entropy-driven elasticity is not trivial, $h k l$-dependent lattice-elasticity has been simulated [50]. There could be either chemical-dependent anisotropy [50] and/or electronic-structure-dependent Piels stress, and thermally activated mechanisms in crystal plasticity [51]. Above all, within elastic deformation range, the metals in a long-range-ordered structure are rarely seen to exhibit such an asymmetric tensor responses as this $\mathrm{CoCrFeMnNi}$ high-entropy alloy does at $473 \mathrm{~K}$ and $673 \mathrm{~K}$. Within $473 \mathrm{~K}$ to $673 \mathrm{~K}$, a crossover of entropy-driven and energy-driven elasticity is observed for the high-entropy alloy. At higher temperature, the severe-distorted-lattice-induced crossover is released. At room temperature, the phenomena is not obvious.

In summary, lattice elasticity of a CoCrFeMnNi high-entropy, a 304L stainless steel medium-entropy alloys, and a pure Ni and a pure Fe low-entropy metals is investigated. Orientation-dependent $h k l$-moduli are calculated. The low-entropy $\mathrm{Ni}$ and Fe, and medium-entropy stainless steel follow Taylor's symmetrical-tensor behavior from room temperature to elevated temperature. For high-entropy equal-molar $\mathrm{CoCrFeMnNi}$ alloy, there is asymmetric lattice-anisotropy responses 
deviating from Taylor's linearity. The long-range-order face-centered-cubic structure of the high-entropy equal-molar CoCrFeMnNi alloy has never changed throughout the measurements. Since there is also no appearance of superlattice peaks during the deformation subjected to temperature changes, there is no order-disorder transition within the high-entropy alloy. The most possible mechanism is the competition between the entropy-driven and the energy-driven elasticity originating from the local heterogeneity.

\section{Acknowledgements}

EWH appreciates the support from Ministry of Science and Technology (MOST) Program 101-2221-E-008-039-MY3 and Atomic Energy Council (AEC) Program 10309037L. Research conducted at ORNL's Spallation Neutron Source was sponsored by the Scientific User Facilities Division, Office of Basic Energy Sciences, US Department of Energy. EWH and his group members very much appreciate the financial support from the National Synchrotron Radiation Research Center (NSRRC) Neutron Program, with Dr. Jason Gardner as NSRRC-Neutron-Program Director.

\section{References}

[1] B. Gludovatz, A. Hohenwarter, D. Catoor, E.H. Chang, E.P. George, R.O. Ritchie, Science, 345 (2014) 1153-1158.

[2] B.S. Murty, J.W. Yeh, S. Ranganathan, High Entropy Alloys, Elsevier Science \& Technology, 2014.

[3] C. Zhu, Z.P. Lu, T.G. Nieh, Acta Materialia, 61 (2013) 2993-3001.

[4] F. Otto, A. Dlouhý, C. Somsen, H. Bei, G. Eggeler, E.P. George, Acta Materialia, 61 (2013) 5743-5755.

[5] M.F. Thorpe, E.J. Garboczi, Physical Review B, 42 (1990) 8405-8417. 
[6] A.R. Denton, N.W. Ashcroft, Physical Review A, 43 (1991) 3161-3164.

[7] D. Shechtman, I. Blech, D. Gratias, J.W. Cahn, Physical Review Letters, 53 (1984) 1951-1953.

[8] A. Georges, G. Kotliar, W. Krauth, M.J. Rozenberg, Rev. Mod. Phys., 68 (1996) 13-125.

[9] M.F. Ashby, Acta Metallurgica, 37 (1989) 1273-1293.

[10] A. Miserez, S.S. Wasko, C.F. Carpenter, J.H. Waite, Nature Materials, 8 (2009) 910-916.

[11] S. Reese, S. Govindjee, Mechanics of Time-Dependent Materials, 1 (1997) 357-396.

[12] H.-J. Butt, M. Kappl, H. Mueller, R. Raiteri, W. Meyer, J. Rühe, Langmuir, 15 (1999) 2559-2565.

[13] U. Seifert, Advances in Physics, 46 (1997) 13-137.

[14] M.J. Buehler, T. Ackbarow, Materials Today, 10 (2007) 46-58.

[15] G. Bao, Journal of the Mechanics and Physics of Solids, 50 (2002) 2237-2274.

[16] C. Bustamante, J. Marko, E. Siggia, S. Smith, Science, 265 (1994) 1599-1600.

[17] M.L. Gardel, J.H. Shin, F.C. MacKintosh, L. Mahadevan, P. Matsudaira, D.A.

Weitz, Science, 304 (2004) 1301-1305.

[18] O. Chaudhuri, S.H. Parekh, D.A. Fletcher, Nature, 445 (2007) 295-298.

[19] S. Siegel, Physical Review, 57 (1940) 537-545.

[20] N.W. Lord, The Journal of Chemical Physics, 21 (1953) 692-699.

[21] M.L. Falk, J.S. Langer, Physical Review E, 57 (1998) 7192-7205.

[22] V. Sánchez-Alarcos, V. Recarte, J. Pérez-Landazábal, E. Cesari, J.

Rodríguez-Velamazán, Entropy, 16 (2014) 2756-2767.

[23] J.-M. Dubois, Nat Mater, 9 (2010) 287-288.

[24] T.C. Hufnagel, R.T. Ott, J. Almer, Physical Review B, 73 (2006) 064204.

[25] H. Wagner, D. Bedorf, S. Küchemann, M. Schwabe, B. Zhang, W. Arnold, K.

Samwer, Nat Mater, 10 (2011) 439-442.

[26] G.I. Taylor, Proceedings of the Royal Society of London. Series A, Containing Papers of a Mathematical and Physical Character, 145 (1934) 362-387.

[27] A. Molinari, G.R. Canova, S. Ahzi, Acta Metallurgica, 35 (1987) 2983-2994.

[28] A. Gali, E.P. George, Intermetallics, 39 (2013) 74-78.

[29] F.J. Wang, Y. Zhang, G.L. Chen, Journal of Alloys and Compounds, 478 (2009) 321-324.

[30] Y.-L. Chen, Y.-H. Hu, C.-A. Hsieh, J.-W. Yeh, S.-K. Chen, Journal of Alloys and Compounds, 481 (2009) 768-775.

[31] Y. Wu, W.H. Liu, X.L. Wang, D. Ma, A.D. Stoica, T.G. Nieh, Z.B. He, Z.P. Lu, Applied Physics Letters, 104 (2014) 051910.

[32] K. An, H. Skorpenske, A. Stoica, D. Ma, X.-L. Wang, E. Cakmak, Metallurgical and Materials Transactions A, 42 (2011) 95-99.

[33] H.M. Ledbetter, R.P. Reed, Journal of Physical and Chemical Reference Data, 2 (1973) 531-618.

[34] R. de Wit, Journal of Applied Crystallography, 30 (1997) 510-511.

[35] T. Gnaupel-Herold, P.C. Brand, H.J. Prask, Journal of Applied Crystallography, 
31 (1998) 929-935.

[36] T. Hutchings, P.J. Withers, T.M. Holden, T. Lorentzen, Introduction to the Characterization of Residual Stress by Neutron Diffraction, Taylor \& Francis, 2005.

[37] G. Sachs, Zur Ableitung einer Fließbedingung, in: Mitteilungen der deutschen Materialprüfungsanstalten, Springer Berlin Heidelberg, 1929, pp. 94-97.

[38] E. Kröner, Acta Metallurgica, 9 (1961) 155-161.

[39] W.A. Good, Physical Review, 60 (1941) 605-609.

[40] A. Rollett, F. Humphreys, G.S. Rohrer, M. Hatherly, Recrystallization and related annealing phenomena, Elsevier, 2004.

[41] N. Rajmohan, Y. Hayakawa, J.A. Szpunar, J.H. Root, Acta Materialia, 45 (1997) 2485-2494.

[42] A. Borbély, J.H. Driver, T. Ungár, Acta Materialia, 48 (2000) 2005-2016.

[43] M. Lugovy, A. Aman, C. Yan, N. Orlovskaya, J. Kuebler, T. Graule, M.J. Reece, M. Dong, A.D. Stoica, A. Ke, J. Appl. Phys., 116 (2014) 013504.

[44] R.P. Feynman, Physical Review, 56 (1939) 340-343.

[45] N. Hansen, R. Mehl, A. Medalist, Metallurgical and Materials Transactions A, 32 (2001) 2917-2935.

[46] G.B. Olson, Science, 277 (1997) 1237-1242.

[47] K. Lu, Science, 328 (2010) 319-320.

[48] F. Roters, P. Eisenlohr, L. Hantcherli, D.D. Tjahjanto, T.R. Bieler, D. Raabe, Acta Materialia, 58 (2010) 1152-1211.

[49] R.O. Ritchie, Nat Mater, 10 (2011) 817-822.

[50] T. Zhu, J. Li, Progress in Materials Science, 55 (2010) 710-757.

[51] D. Caillard, J. Martin, Thermally activated mechanisms in crystal plasticity, Cambridge Univ Press, 2003. 


\section{Figure captions:}

\section{Figure 1.}

Variation of reciprocal moduli subjected to anisotropy factor for (a) pure $\mathrm{Ni}$, (b) pure Fe, (c) 304L stainless steel, and (d) CoCrFeMnNi HEA.

\section{Figure 2.}

Lattice moduli evolution as a function of temperature for (a) pure $\mathrm{Ni}$, (b) pure $\mathrm{Fe}$, (c) 304L stainless steel, and (d) CoCrFeMnNi HEA. 

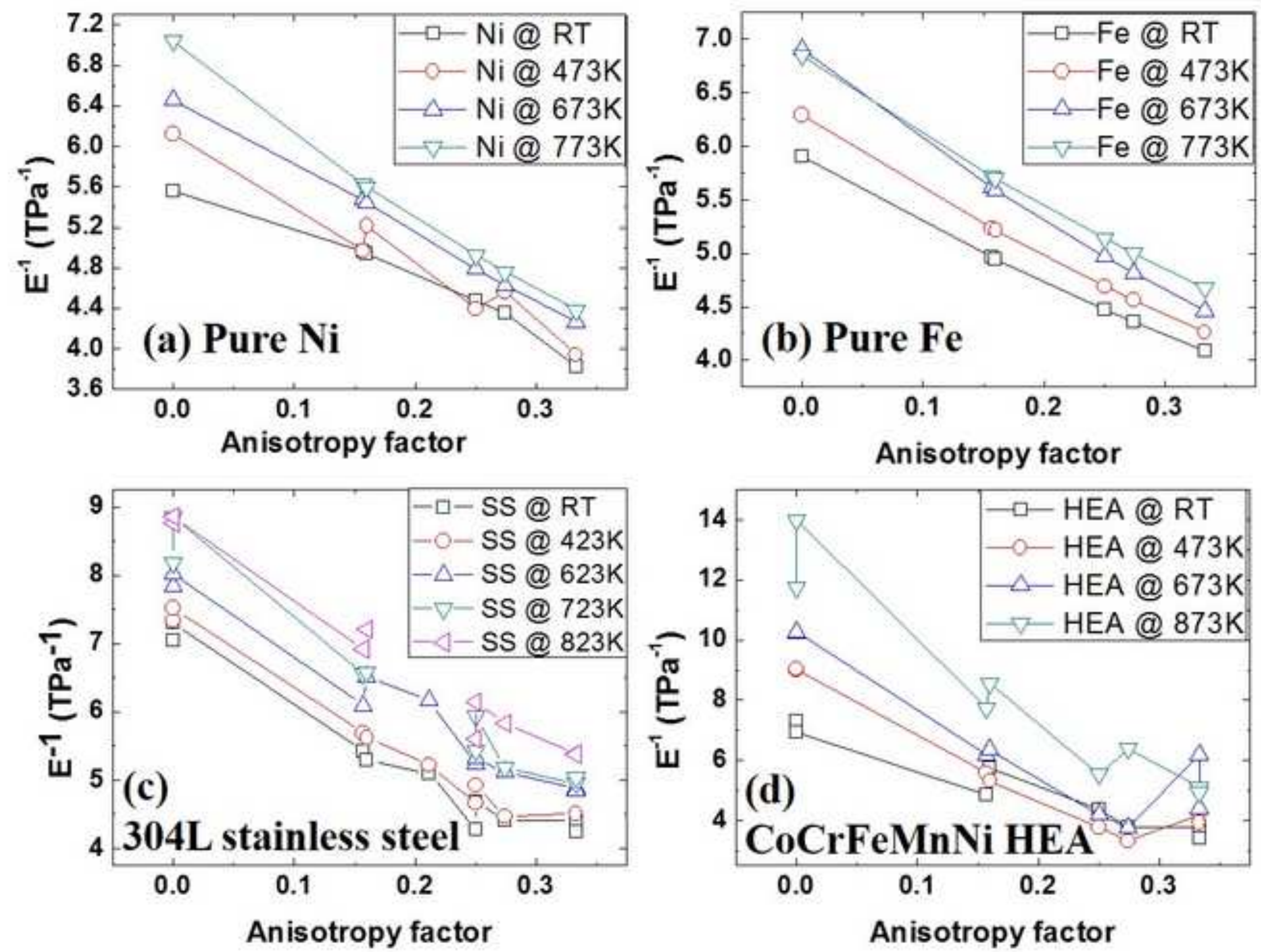

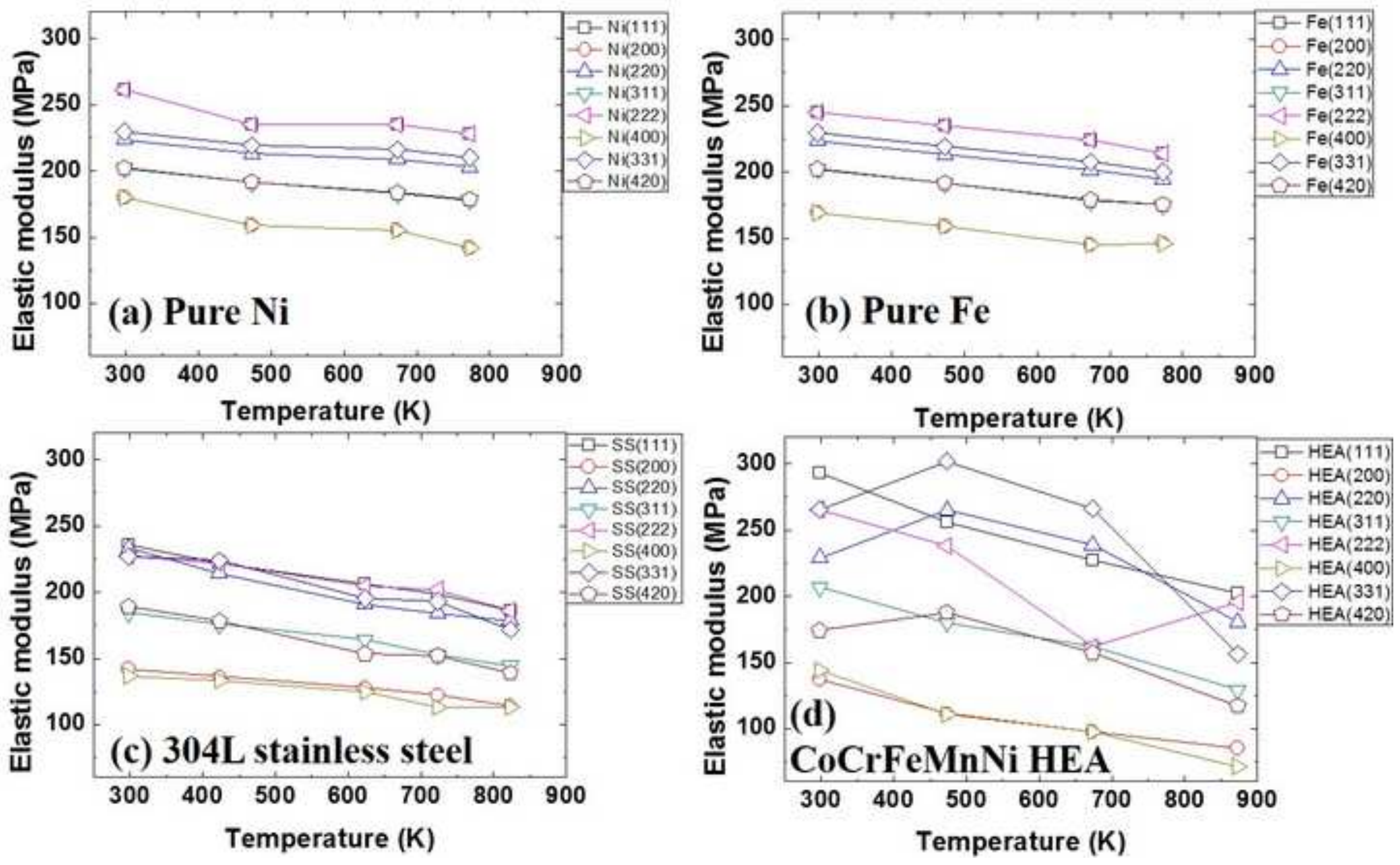